\title{
Specific Resistance Resources in the Salutogenic Model of Health
}

\author{
Maurice B. Mittelmark, Marguerite Daniel, \\ and Helga B. Urke
}

\section{Introduction}

This chapter discusses conceptual and concrete differences between generalized and specific resistance resources in the salutogenic model of health. How generalized resistance resources (GRRs) and specific resistance resources (SRRs) are developed differ, with implications for health promotion practice.

To summarize, the main idea is that GRRs arise from the cultural, social, and environmental conditions of living and early childhood rearing and socialization experiences, in addition to idiosyncratic factors and chance (Lamprecht \& Sack, 2003; Lindström \& Eriksson, 2005). SRRs, on the other hand, are optimized by societal action in which health promotion has a contributing role, for example, the provision of supportive social and physical environments.

\section{The Salutogenic Model of Health Logic}

Antonovsky (1987) called for research to develop scientific knowledge about strengthening the sense of coherence. This could be done by building on the resistance resources (RR) that are the properties of individuals, groups, and even situations. GRRs facilitate coping with stressors and strengthen the sense of coherence. Confronting the question of how a strong sense of coherence translates into better health, Antonovsky proposed that "a strong $\mathrm{SoC}[\ldots]$ allows one to 'reach out,' in any given situation, and apply the resources appropriate to that stressor" (Antonovsky, 1996, p. 15).

This chapter is a revision and update of work published in Mittelmark, M.B., Sagy, S., Eriksson, M., Bauer, G., Pelikan, J.M., Lindström, B., \& Espnes, G.A. (eds). (2017). The Handbook of Salutogenesis. Springer, Cham. DOI: https://doi.org/10.1007/978-3-319-04600-6.

M. B. Mittelmark $(\varangle) \cdot$ M. Daniel $\cdot$ H. B. Urke Department of Health Promotion and Development, Faculty of Psychology, University of Bergen, Bergen, Norway

e-mail: maurice.mittelmark@uib.no
The highly simplified salutogenic model of health logic is

$$
\mathrm{RR} \rightarrow \uparrow \mathrm{SOC} \rightarrow \uparrow \text { use of } \mathrm{RR} \rightarrow \uparrow \mathrm{HEALTH}
$$

GRRs and SRRs will be formally defined later, but for now, GRRs are resources that have wide-ranging utility (one's social network, for example). In contrast, SRRs have situation-specific utility (e.g. an emergency phone number to reach the police). Antonovsky felt it was

\footnotetext{
...imperative to focus on developing a fuller understanding of those generalized resistance resources that can be applied to meet all demands (Antonovsky, 1972, p. 541), while
}

... [SRRs] are often useful in particular situations of tension. A certain drug, telephone lifelines of suicide prevention agencies... can be of great help in coping with particular stressors. But these are all too often matters of chance or luck, as well as being helpful only in particular situations...[and] ... it is the GRR that determines the extent to which specific resistance resources are available to us (Antonovsky, 1979, p. 98-99).

This goes a great way toward explaining why Antonovsky's attention was mostly on the left side of this more detailed salutogenic model of health diagram:

$$
\begin{aligned}
& \text { GRR } \rightarrow \uparrow \mathrm{SOC} \rightarrow \uparrow \text { use of GRR } \\
& \& \uparrow \text { use of SRR } \rightarrow \uparrow \text { HEALTH }
\end{aligned}
$$

A more realistic depiction would be a systems-like diagram with double-headed arrows connecting everything to everything. The simplification above is useful for the present purpose, which is to elucidate the GRR/SRR distinction.

SRRs need not always be "matters of chance or luck". Indeed, it is an essential aspect of health promotion to replace chance and luck with fair and dependable availability of SRRs that support health. One of the highest priorities of health promotion is providing supportive environments for health (WHO, 2009). Supportive environments include both GRRs and SRRs, but as suggested in the salutogenic model of health logic above, they have distinctions.

Most of the space in this chapter is devoted to discussing the nature of SRRs and health promotion's role in their 
nurturance. However, some space is given to a brief overview of the nature of GRRs, to help illuminate distinctions, similarities, and interrelationships between GRRs and SRRs. The reader interested in a full exposition of GRRs is referred to Chap. 12 in this handbook and to chapter 4 in Antonovsky's Health, Stress and Coping Antonovsky (1979).

Antonovsky (1979, P. 99) defined a GRR as "any characteristic of the person, the group, or the environment that can facilitate effective tension management". He was quite clear that GRRs and SRRs are not exchangeable concepts: “...it is the GRR that determines the extent to which specific resistance resources are available to us... being literate or being rich... opens the way to exploitation of many specific resistance resources..." (Antonovsky, 1979, pp. 99-100). A perhaps more precise formulation is that when confronted with a particular stressor, a strong sense of coherence enhances one's ability to recognize and activate the most appropriate SRR from those that may be available.

Antonovsky (1979, pp. 103-119) discussed GRRs that operate through physical and biochemical mechanisms (e.g. immune function) that enable the acquisition of SRRs (as money may do), that are intrapersonal (e.g. with ego identity, intelligence, and coping), that are social (interpersonal ties and social embeddedness), and that are cultural (guiding as to how stressors should be encountered). GRRs play two important roles in coping: they help determine the strength of the sense of coherence, and they enable the use of specific resistance resources.

\section{Specific Resistance Resources}

Neither Antonovsky nor the few others who have written about SRRs have shown much interest in the GRR/SRR differentiation. For example, in Antonovsky's (1979) extremely detailed depiction of the salutogenic model of health (ibid, pp. 184-185), a strong sense of coherence is shown as mobilizing GRRs and SRRs, with no clear differentiation of the two. Both types of resistance resources are posited to have roles in avoiding stressors, in the definition of stimuli and non-stressors, and in overcoming stressors. Antonovsky hardly mentioned SRRs in his Unraveling the Mystery of Health, and he did not dwell on the distinction between GRRs and SRRs:

What the person with the strong SoC does is ... [choose] from the repertoire of generalized and specific resistance resources at his or her disposal... (Antonovsky, 1987, p. 138).

Others seem to agree that the GRR/SRR distinction is not particularly important. Poppius (2007) wrote about choosing "from the repertoire of generalized and specific resistance resources [...] in what seems to be the most appropriate combination". Nene (2006) noted that the sense of coherence is influenced by GRRs and SRRs and makes no differentiation between them. Sullivan (2006) does make a differentiation, stating that nursing is a GRR while the nurse providing help with a particular problem is an SRR. Yet, Sullivan does not develop that distinction in terms of the role of sense of coherence. Haldeman and Peters (1988) intended to measure SRRs in a study to identify the combination of SRRs and tension that would best predict stress. They operationalized SRRs as satisfaction with family life and family finances, frequency of interactions with friends and relatives, and community resources used. These measures are distant from the concept of SRRs as distinguished from GRRs, even if the number of community resources is measured. SRRs are particular resources used in encounters with particular stressors, as in Antonovsky's example of using a suicide hotline by a suicidal person. Reininghaus et al. (2007) noted the distinction between GRRs and SRRs, in a study of the stress connected to assault on psychiatric nurses, and then rejected the distinction by creating a measure of "stress resistance resources" composed of self-esteem (a GRR), self-confidence (a GRR), received clinical supervision (an SRR), and staff support services (an SRR). Taylor (2004) differentiated GRRs and SRRs in her literature review of salutogenesis as a framework for child protection, but characterized both, without differentiation, as helping people to structure life experiences to reinforce the sense of coherence.

These citations are not "cherry-picked", highly selected counter-examples from an extensive literature in which GRRs and SRRs are discussed: they are all the instances in which SRRs received explicit attention in a reasonably thorough literature search.

Why do SRRs receive so little attention? One answer is that following Antonovsky's lead, there has been all-consuming attention to GRRs and the sense of coherence (Eriksson \& Lindström, 2005) and its relationship to health and well-being (Eriksson \& Lindström, 2006, 2007). Even if Antonovsky wished health promotion to focus on the sense of coherence as the dependent variable, most researchers have focussed on it as the independent variable. While this could be assumed to drive interest in SRRs as mediators in the sense of coherence/health relationship, such interest is not manifest. On the contrary, there has been little interest in the question of what mediates the connection between the sense of coherence and health, despite Antonovsky's postulation that a strong sense of coherence allows one, in any given situation, to apply the appropriate GRR and/or SRR (Antonovsky, 1979).

One additional, critical point needs to be made to clarify why SRRs have received little attention in salutogenic research and why this should be rectified. As already noted, Antonovsky viewed SRRs as all too often matters of chance or luck. In the mid-1990s, he observed that health promotion had not: 
...confronted the question of the creation of the appropriate social conditions which underlie or facilitate health-promotive behaviors, for example adequate day care facilities and access to health care, not to speak of incomes adequate for decent nutrition and housing. (Antonovsky, 1996, p. 12)

Put in contemporary terms, Antonovsky referred to social determinants of health (e.g. the GRR "income") and supportive social environments (e.g. the SRR "daycare facilities"). Examined closely, what may be a GRR from one person's perspective may be an SRR from another person's perspective. For example, a child daycare facility is an SRR for the parents, and hopefully, a source of GRRs for the child. In contrast, an eldercare facility is an SRR for the resident and an SRR for relatives that need professional care assistance.

Antonovsky's criticism of health promotion for not confronting social conditions was perhaps valid for the approach to health promotion that dominated in Europe and the USA in the 1970s and 1980s, concerned mostly with individuals' responsibility for their health and calling for individuals to abandon their risk behaviour to prevent chronic diseases.

However, health promotion has evolved. The 1986 Ottawa Charter for Health Promotion acknowledged individuals' responsibility but emphasized the importance of social determinants of health and creating supportive environments (Eriksson \& Lindström, 2008; Kickbusch, 2003). In recent decades, health promotion moved from an almost myopic concern with individuals' health-related lifestyles to a balanced concern with processes for empowering individuals and communities to control their health. In good part, this is accomplished by creating environments supportive of health, or "appropriate social conditions" in Antonovsky's words. Health promotion's concern with appropriate social conditions has taken two forms. One is an overarching emphasis on reducing social inequities in health by a fairer distribution of social resources (Marmot et al., 2008). The other is the health promotion "settings" approach, in which schools, workplaces, and whole communities are considered as locales for health promotion, expanding from the traditional locus of health care in doctors' offices, health clinics, and hospitals (Dooris et al., 2007; Poland et al., 2009). Does health promotion's settings approach mean that it has engaged the SRR concept or the GRR concept? A nuanced answer depends in part on a precise definition of specific resistance resources.

\section{Definition of Specific Resistance Resources}

A useful definition of SRRs must distinguish them from GRRs. Bengt Lindström is famous for his illustrated lectures on salutogenesis. A cartoon figure travels across the chalkboard, in the river of life, encountering stressors, trials, and tribulations, equipped with a knapsack stuffed with GRRs acquired during a lifetime (Fig. 13.1). The main point is that the GRRs are already available to be engaged as needed as one encounters various situations creating tension. In concert

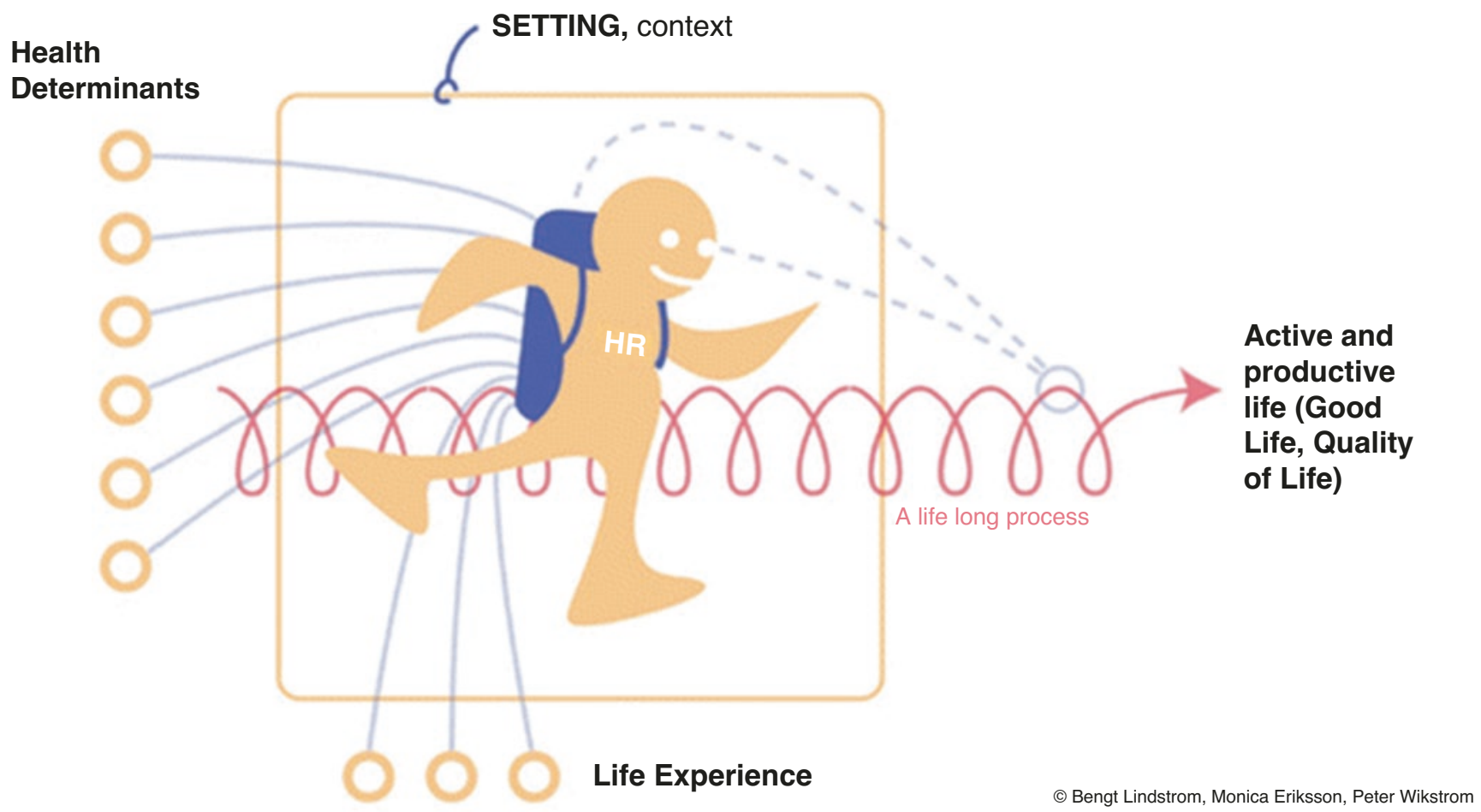

Fig. 13.1 Generalized resistance resources in the knapsack. (@ Bengt Lindström, Monica Eriksson, Peder Wikström. All Rights Reserved) 
with this metaphor, we conceptualize SRRs as available in the river, picked up and used as needed in specific encounters with stressors, and not necessarily placed in the knapsack afterwards. The relationship between GRRs and SRRs is that via the sense of coherence, GRRs enable one to recognize, pick up, and use SRRs in ways that keep tension from turning into debilitating stress, assuming useful SRRs are available.

A brief example: Having access to and understanding the empowering potential of the Internet is a GRR. The availability of information about your present worrying symptoms on Wikipedia is an SRR. That you have access to, and proactively search for, read, critically evaluate, and use the Wiki's information exemplifies the salutogenic model of health logic:

\section{(a) GRR $\rightarrow \uparrow$ use of SRR $\rightarrow \uparrow$ HEALTH \\ (b) INTERNET $\rightarrow \uparrow$ SPECIFIC WIKI $\rightarrow \uparrow$ HEALTH}

This is, of course, an oversimplification. For example, while the Internet has undoubtedly contributed to an enhanced sense of coherence for many people, it is but one of many GRRs having an equal or more significant influence on the sense of coherence. The diagram's point is not to depict the salutogenic model of health in detail but to show how GRRs and SRRs are substantially different. Of course, health promotion interventions might focus on both-increasing people's unfettered access to the Internet and their skill in using it (enhanced GRR) and making websites that address various specific health issues that are of salience when particular nasty symptoms pop up (enhanced SRR).

A formal definition of SRRs is shown in Fig. 13.2, using Facet Theory's sentence mapping approach (Borg \& Shye, 1995; Canter, 2012). Antonovsky (1979) used the same approach to define key concepts, including health on the ease/dis-ease continuum (ibid, p. 65), GRRs (ibid, p. 103), and the sense of coherence (Antonovsky, 1987, p. 77). The elements in the three arrays of the mapping sentence definition are not meant to be exhaustive but rather are illustrative.

SRRs are instrumentalities whose meanings are defined in terms of the particular stressors they are invoked to manage.

A GRR is a generality, and an SRR is a particularity.

Usually, SRRs are not invoked unless tension is perceived to threaten to convert to debilitating stress, which many tensions do not. The salutogenic model of health is concerned with ubiquitous tensions that can convert to health-threatening levels of stress. Antonovsky (1979, pp. 89-90) listed these:

\begin{abstract}
... accidents and the survivors; the untoward experiences of others in our social networks; the horrors of history in which we are involved; intrapsychic, unconscious conflicts and anxieties; the fear of aggression, mutilation and destruction; the events of history brought into our living rooms; the changes of the narrower world in which we live, phase-specific psychosocial crises; other normative life crises-role entries and exits; inadequate socialization, underload, and overload; the inherent conflicts in all social relations; and the gap between culturally inculcated goals and socially structured means.
\end{abstract}

A useful examination of the differences between GRRs and SRRs should be undertaken with this understanding of psychosocial stressors in mind. At extremes-surviving a plane crash, taking an exam-stressors are stressors from the start or tensions that simply remain tensions.
Fig. 13.2 Mapping sentence definition of specific resistance resources. (Reproduced with permission from Mittelmark et al. (2017))

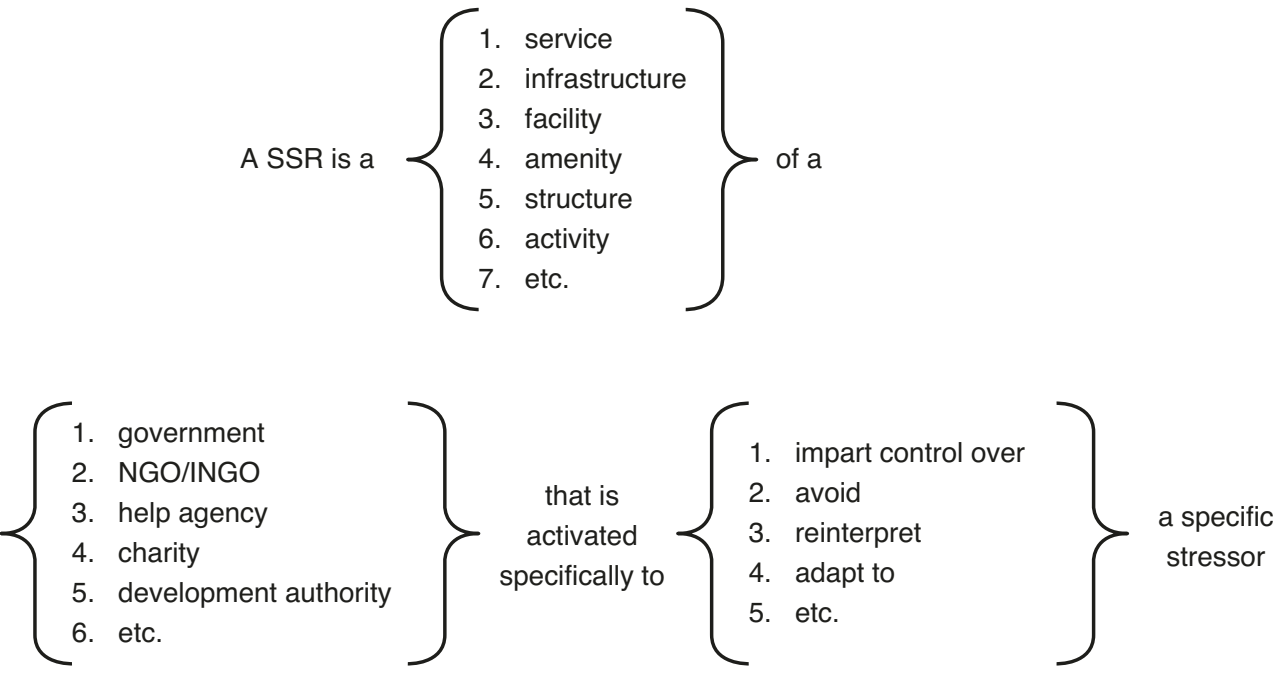

and thus prevent tension from being transformed into stress. 


\section{Examples of Specific Resistance Resources}

\section{Health-Promoting Schools in Norway}

Virtually all adolescents attending school are stressed by the demands of maturation, peer relations, teachers' demands, home and community conditions, and so on. In this cauldron of tension, schools in Norway (and in many other countries) have implemented Health Promoting Schools programmes which aim to support educational goals by attending to the health and well-being of all who inhabit the school milieu; students, teachers, administrators, parents, and neighbourhoods (Langford et al., 2015; Tjomsland et al., 2009; Wold \& Mittelmark, 2018). The health-promoting school strives to meet these goals:

- Promote the health and well-being of students.

- Enhance the learning outcomes of students.

- Uphold social justice and equity concepts.

- Provide a safe and supportive environment.

- Promote student participation and empowerment.

- Link health and education issues and systems.

- Address the health and well-being issues of all school staff.

- Collaborate with parents and the local community.

- Integrate health into the school's ongoing activities, curriculum, and assessment standards.

- Set realistic goals built on accurate data and sound scientific evidence.

- Seek continuous improvement through ongoing monitoring and evaluation.

Thus, the health-promoting school aims to be a powerful GRR for the youth, the staff, the parents, and the surrounding community. The Norwegian school is also a repository of, or a portal to, some SRRs, offered through the school nursing service (Moen \& Skundberg-Kletthagen, 2018) and other student-support services. Examples include support for pregnant students to help keep them in school, special education teachers and facilities equipped to help students with learning disabilities, and referral to community child protection services in cases of need.

These SRRs are present in or around the school, but they are not particularly salient to the adolescents that do not need them, and therefore do not use them. The school as a GRR helps contribute in a general way to strengthen the sense of coherence of many pupils, and a strong sense of coherence facilitates the uptake/use of particular SRRs when the need should arise. Let us consider two students. Jack has a typical day, experiencing "normal" strain and hassles, but nothing out of the ordinary happens. SRRs abound, but this student makes use of none of them; they are not salient. On the same day, Jill discovers she is pregnant, and her sense of coher- ence is high enough that she does not panic and sink into depression. Instead, she contacts the pregnancy support programme, which she knows about and trusts because of other pupils' good experiences. The pregnancy support programme is an SRR for this student, offering highly salient services at this inflection point in her life.

There is a vexing equity dimension to this. If SRRs are more readily available to those with lots of GRRs (e.g. money), SRRs might contribute to a widened equity gap. Equality in access to SRRs depends on a reasonably fair distribution of GRRs, so health promotion needs to focus on both types.

\section{Child Health in the Andean Highlands in Peru}

Perhaps the most apparent SRRs are social services established to provide targeted assistance to groups living in conditions that impart severe vulnerability (Mittelmark, 2021). A prime example was documented in research in a remote and low-income district in the Andean highlands in Peru (Urke et al., 2013). The field researcher (Urke) interviewed mothers with local reputations for providing good childcare. All the mothers participated in a Peruvian NGO-run social and health programme. The interviewer did not mention the NGO programme - a clear example of a social services SRR - to avoid prompting. The respondents explicitly referred to it and attributed improved health-related knowledge and skills to the NGO interventions that included education about nutritious meal preparation, childcare skills, and sanitation practices. This project also illuminated the close relation between GRRs and SRRs. There was some evidence that the women with more GRRs (such as money and social support in the home and the community) benefited more from the NGO's activities than did the women with fewer GRRs.

\section{Support Services for Orphaned Children and Adolescents in Botswana}

Another example of NGO-as-SRR is described in research from Botswana (Thamuku \& Daniel, 2012). In collaboration with the Botswana Department of Social Services, the Botswanan NGO People and Nature Trust developed a programme called Ark for Children to provide support services for orphaned children and adolescents. They employ culturally appropriate interventions to strengthen orphans and build them into cohesive groups of age-mates who support each other. Ark for Children organizes 16-day retreats for up to 40 adolescent orphans from the same village. The retreats harken to historical Setswana initiation rites and link with cultural traditions recognized by the members of the adoles- 
cents' community. The adolescents are followed up in a 3-year support programme. This is an illuminating example of an SRR (Arkfor Children) that builds GRRs (social cohesion and supportive ties).

\section{Sexual Orientation and Gender-Identity Support Services in the USA}

Identity-based adversity begins early in life, affecting the availability and development of GRRs. Young people's experience of sexual orientation prejudice is exemplified in many cases by bullying, harassment, rejection, social isolation, and internalization of negative attitudes and beliefs. Genderidentity adversity is framed by stigma arising from societal norms and biases (Horn, 2019). While sexual preferencerelated adversity is typically an emergent feature in adolescence and young adulthood, gender-role tension may have roots in earliest childhood behaviour. Little boys are encouraged to dress, think, and act like boys "should" and little girls to act like girls, with norms varying according to their culture. Boys holding hands beyond young childhood is not gender-consistent in many cultures but is normative in other cultures. This points out that salutogenesis' relevance to identity-based adversity, tension, and coping is complex, rooted in culture and impacting one's early life experience, the acquisition of GRRs, the availability and use of SRRs, and therefore the development of the SOC.

In this chapter on the nature of SRRs, we turn aside momentarily, noting that the experience of and developing resilience to gender-related prejudice may be a GRR. In their research with young adults in the USA, Schmitz and Tyler's (2018) respondents explained it in ways like this: "[My lesbian identity] opens up a lot more doors... I think that [it] opens up opportunities for friendship because you like to confide in people that have similar situations, so I think it builds stronger relationships".

Now narrowing the discussion to SRRs, we focus on just one dimension of salutogenesis and gender-based identity: the tension, stress, and coping-enabling SRRs of young persons with lesbian, gay, bisexual, transgender, and queer (LGBTQ ${ }^{1}$ ) identities. In coping research with lesbian, gay, and bisexual (LGB) American Latino and non-Latino young adults, Toomey et al. (2018) examined the use of three types of coping strategies: engagement of SRRs, alternatives-seeking strategies (e.g. finding new friends), and cognitive strategies (e.g. imagining a better future). Regarding SRRs, respondents reported employing a closely knit constellation

${ }^{1}$ Some of the acronyms used below vary from this form, following the usages of the various authors cited. of coping behaviours, including getting involved in LGBT groups and organizations, looking for services for LGBT youth, and looking for information on LGBT issues.

Similar findings are reported by Asakura (2016), whose LGBTQ respondents reported coping by engaging in collective action, by participating in safe social spaces like gaystraight alliances, and by accessing social services from organizations that welcome gender and sexual diversity among youth. Likewise, Schmitz and Tyler (2018) observed the importance of institutional support in LGBT coping. As one college student expressed it, "[the campus] is very open and accepting... there are plenty of resources on campus, specifically for LGBT students".

\section{Non-institutional SRRs in Caregiving Dyads in Sweden}

Figure 13.2 may convey an impression that SRRs are exclusively facilities and organizations whose mission is to provide specific types of assistance to people with particular needs. Yet, the definition does embrace the concept of SRRs in the private sphere ("activities", listed sixth in the first facet of the definition). This has been well described in a Swedish programme of research with caregiving dyads in which care was provided to persons aged 65+ (Wennerberg et al., 2016). Caregivers' overall feelings of competence in being a caregiver-a GRR in the researchers' analysis-were bolstered by caregiver SRRs such as having enough physical strength to provide physically demanding care activities (e.g. lifting) and having the professional/technical knowledge required for managing complex medication regimes. In these caregiving dyads, having children and grandchildren functioned as an SRR, a shared experience that was a mutual pleasure to the dyad (Wennerberg et al., 2019). A mutual understanding of the caregiving situation and good dyad communication skills was also revealed as an important SRR (ibid). For dyads in assisted living facilities, the facility - an SRRenabled caregivers to gain/regain personal SRRs that had been problematic in their homes (SRR deficits). Examples cited by respondents included shopping facilities in the living complex, elevators, and apartments that were disability adapted (Eriksson et al., 2017).

The Swedish studies cited above also report findings regarding general and specific resources deficits (GRDs and SRDs) (Wennerberg et al., 2019). A discussion of GRDs and SRDs is beyond this chapter's scope. Still, it is in place to mention that the general-specific differentiation also pertains to negative life experiences, unsuccessful tension management, and a weakened sense of coherence. A caregiver's loss of a well-functioning partner is simultaneously a GRD-the 
fading quality and equality of a mutually caring and sharing relationship_and an SRD as in the care receiver's reduced ability to reciprocate care (ibid).

\section{Conclusion}

This chapter's aim has been modest, to illuminate a part of the salutogenic model of health that seems to be overlooked-SRRs have as much or more relevance to health promotion practice as do GRRs. By drawing attention to SRRs, one also draws attention to what should be a core aim of health promotion: to ensure that the availability of the right SRR at the right time is not all too often a matter of "chance or luck", as Antonovsky worried.

\section{References}

Antonovsky, A. (1972). Breakdown. A needed fourth step in the conceptual armamentarium of modern medicine. Social Science and Medicine, 6, 537-544.

Antonovsky, A. (1979). Health, stress and coping. Jossey-Bass.

Antonovsky, A. (1987). Unraveling the mystery of health-How people manage stress and stay well. Jossey-Bass.

Antonovsky, A. (1996). The salutogenic model as a theory to guide health promotion. Health Promotion International, 11(1), 11-18.

Asakura, K. (2016). Paving pathways through the pain: A grounded theory of resilience among lesbian, gay, bisexual, trans and queer youth. Journal of Research on Adolescence, 27(3), 521-536.

Borg, I., \& Shye, S. (1995). Facet theory: Form and content. Sage.

Canter, D. (Ed.). (2012). Facet theory: Approaches to social research. Springer.

Dooris, M., Poland, B., Kolbe, L., De Leeuw, E., McCall, D. S., \& Wharf-Higgins, J. (2007). Healthy settings. In D. V. McQueen \& C. M. Jones (Eds.), Global perspectives on health promotion effectiveness (pp. 327-352). Springer.

Eriksson, M., \& Lindström, B. (2005). Validity of Antonovsky's sense of coherence scale: A systematic review. Journal of Epidemiology and Community Health, 59(6), 460-466.

Eriksson, M., \& Lindström, B. (2006). Antonovsky's sense of coherence scale and the relation with health: A systematic review. Journal of Epidemiology and Community Health, 60(5), 376-381.

Eriksson, M., \& Lindström, B. (2007). Antonovsky's sense of coherence scale and its relation with quality of life: A systematic review. Journal of Epidemiology and Community Health, 61(11), 938-944.

Eriksson, M., \& Lindström, B. (2008). A salutogenic interpretation of the Ottawa Charter. Health Promotion International, 23(2), 190-199.

Eriksson, M., Wennerberg, M. M. T., Lundgren, S. M., \& Danielson, E. (2017). "Self-employed" in caregiving hood: The contribution of Swedish informal caregivers' environmental and contextual resistance resources and deficits. Societies, 7(3), 19. https://doi. org/10.3390/soc7030019

Haldeman, V. A., \& Peters, J. M. (1988). Using resistance resources to reduce stress: A study of rural Nevadans. Journal of Family and Economic Issues, 9(4), 357-366.
Horn, S. S. (2019). Sexual orientation and gender identity-based prejudice. Child Development Perspectives, 13(1), 21-27.

Kickbusch, I. (2003). The contribution of the World Health Organization to a new public health and health promotion. American Journal of Public Health, 93(3), 383-388.

Lamprecht, F., \& Sack, M. (2003). Vulnerability and salutogenesis in health and disease. Public Health Reviews, 31(1), 7-21.

Langford, R., Bonell, C., Jones, H., Pouliou, T., Murphy, S., Waters, E., ... Campbell, R. (2015). The World Health Organization's Health Promoting Schools framework: A Cochrane systematic review and meta-analysis. BMC Public Health, 15(1), 130.

Lindström, B., \& Eriksson, M. (2005). Salutogenesis. Journal of Epidemiology and Community Health, 59(6), 440-442.

Marmot, M., Friel, S., Bell, R., Houweling, T. A., Taylor, S., \& Commission on Social Determinants of Health. (2008). Closing the gap in a generation: Health equity through action on the social determinants of health. The Lancet, 372(9650), 1661-1669.

Mittelmark, M. B. (2021). Resilience in the salutogenic model of health. In M. Ungar (Ed.), Multisystemic resilience: Adaptation and transformation in changing. Springer.

Mittelmark, M. B., Bull, T., Daniel, M., \& Urke, H. (2017). Specific resistance resources in the salutogenic model of health. In M. B. Mittelmark, S. Sagy, M. Eriksson, G. Bauer, J. M. Pelikan, B. Lindström, \& G. A. Espnes (Eds.), The handbook of salutogenesis. Springer International Publishing.

Moen, Ø. L., \& Skundberg-Kletthagen, H. (2018). Public health nurses' experience, involvement and attitude concerning mental health issues in a school setting. Nordic Journal of Nursing Research, 38(2), 61-67.

Nene, N. (2006). Compassion fatigue and the sense of coherence in caregivers working with sexually abused children in Soweto. Doctoral dissertation, University of the Witwatersrand, Johannesburg.

Poland, B., Krupa, G., \& McCall, D. (2009). Settings for health promotion: An analytic framework to guide intervention design and implementation. Health Promotion Practice, 10(4), 505-516.

Poppius, E. S. K. O. (2007). The sense of coherence and health. The effects of the sense of coherence on risk of coronary heart disease, cancer, injuries and all-cause mortality. Tampereen yliopisto.

Reininghaus, U., Craig, T., Gournay, K., Hopkinson, P., \& Carson, J. (2007). The high secure psychiatric hospitals' nursing staff stress survey 3: Identifying stress resistance resources in the stress process of physical assault. Personality and Individual Differences, 42(3), 397-408.

Schmitz, R. M., \& Tyler, K. A. (2018). 'Life has actually become more clear': An examination of resilience among LGBTQ young adults. Sexualities, 22(4), 710-733.

Sullivan, G. C. (2006). Evaluating Antonovsky's Salutogenic Model for its adaptability to nursing. Journal of Advanced Nursing, 14(4), $336-342$.

Taylor, J. S. (2004). Salutogenesis as a framework for child protection: Literature review. Journal of Advanced Nursing, 45(6), 633-643.

Thamuku, M., \& Daniel, M. (2012). The use of rites of passage in strengthening the psychosocial well-being of orphaned children in Botswana. African Journal of AIDS Research, 11(3), 215-224.

Tjomsland, H. E., Iversen, A. C., \& Wold, B. (2009). The Norwegian network of health promoting schools: A three-year follow-up study of teacher motivation, participation and perceived outcomes. Scandinavian Journal of Educational Research, 53(1), 89-102.

Toomey, R. B., Ryan, C., Diaz, R. M., \& Russell, S. T. (2018). Coping with sexual orientation-minority stress. Journal of Homosexuality, 65(4), 484-500.

Urke, H. B., Bull, T., \& Mittelmark, M. B. (2013). Child diet and healthy growth in the context of rural poverty in the Peruvian Andes: What 
influences primary caregivers' opportunities and choices? Global Health Promotion, 20(3), 5-13.

Wennerberg, M. M. T., Eriksson, M., Danielson, E., \& Lundgren, S. M. (2016). Unravelling Swedish caregivers' generalized resistance resources. Scandinavian Journal of Caring Sciences, 30, 602-613.

Wennerberg, M. M. T., Lundgren, S. M., Eriksson, M., \& Danielson, E. (2019). Me and you in caregivinghood - Dyadic resistance resources and deficits out of the informal caregiver's perspective. Aging \& Mental Health, 23(8), 1041-1048.
Wold, B., \& Mittelmark, M. B. (2018). Health-promotion research over three decades: The social-ecological model and challenges in implementation of interventions. Scandinavian Journal of Public Health, 46(20_suppl), 20-26.

World Health Organization. (2009). Milestones in health promotion. Statements from global conferences. WHO Press, World Health Organization.

Open Access This chapter is licensed under the terms of the Creative Commons Attribution 4.0 International License (http://creativecommons. org/licenses/by/4.0/), which permits use, sharing, adaptation, distribution and reproduction in any medium or format, as long as you give appropriate credit to the original author(s) and the source, provide a link to the Creative Commons license and indicate if changes were made.

The images or other third party material in this chapter are included in the chapter's Creative Commons license, unless indicated otherwise in a credit line to the material. If material is not included in the chapter's Creative Commons license and your intended use is not permitted by statutory regulation or exceeds the permitted use, you will need to obtain permission directly from the copyright holder. 